\title{
Region-Based 2D Deformable Generalized Cylinder for Narrow Structures Segmentation
}

\author{
Julien Mille ${ }^{1}$, Romuald Boné ${ }^{1}$, and Laurent D. Cohen ${ }^{2}$ \\ ${ }^{1}$ Laboratoire d'Informatique, Université François Rabelais de Tours \\ 64 avenue Jean Portalis, 37200 Tours, France \\ ${ }^{2}$ CEREMADE, CNRS UMR 7534, Université Paris Dauphine \\ Place du Maréchal de Lattre de Tassigny, 75775 Paris, France \\ julien.mille@univ-tours.fr
}

\begin{abstract}
In this paper, we present a region-based deformable cylinder model, extending the work on classical region-based active contours and gradient-based ribbon snakes. Defined by a central curve playing the role of the medial axis and a variable thickness, the model is endowed with a region-dependent term. This energy follows the narrow band principle, in order to handle local region properties while overcoming limitations of classical edge-based models. The energy is subsequently transformed and derived in order to allow implementation on a polygonal line deformed with gradient descent. The model is used to extract path-like objects in medical and aerial images.
\end{abstract}

\section{Introduction}

In order to segment objects with respect to region homogeneity criterion, a large family of region-based active contour models has been developed over the last decade. Extending the initial boundary-based snake by Kass et al [1, regionbased active contours exhibit desirable property of holding global features about the region of interest 2. Parametric implementations include the anticipating snake [3], the statistical snake [4 and the mixed model of [5] whereas implicit contours based on the level set framework include the active contours without edges [6], region descriptors [7] and the geodesic active regions [8].

The problem of recovering narrow structures using deformable models was addressed for instance in [9] in the context of cortex segmentation. The ribbon snake model [10] 11] [12, inspired by the work in [13], was developed for road extraction in aerial images. A different kind of model was proposed recently for vessels in [14. By its geometrically constrained formulation, the ribbon snake model is well suited to extract path-like objects. Initial ribbon snakes are boundary-based, since they attach the model by means of a gradient-dependent term integrated over the contour. In presence of images suffering from noise and lack of salient boundaries between neighbouring structures, embedding a region term in the energy functional allows to describe homogeneity over the whole shape.

As a result, we extend the ribbon snake, which is actually a $2 \mathrm{D}$ generalized cylinder (GC), to region-based segmentation. Our GC is made up of a central

D. Forsyth, P. Torr, and A. Zisserman (Eds.): ECCV 2008, Part II, LNCS 5303, pp. 392 404, 2008.

(C) Springer-Verlag Berlin Heidelberg 2008 
planar curve, acting as a medial axis, and a variable thickness. This defines a band over which a region energy is formulated. Region-based active contours usually consider both inner and outer statistics 6 . Similarly, we use the narrow band principle [15] to define outer regions. The GC is subsequently deformed by coupled evolution of the curve and the thickness guided by energy minimization. When dealing with region integrals, the combined use of Green-Riemann theorem and calculus of variations enables to derive the Euler-Lagrange equations. Moreover, the minimal path approach of Cohen and Kimmel [16] provides a reliable initialization for the $\mathrm{GC}$, preventing it from being trapped in erroneous local minima.

The paper is organized as follows. Section 2 presents the 2D deformable GC, describing its geometrical formulation and its associated energies. The model has a particular shape, which allows transformation of the region integral leading to a suitable form for polygonal implementation. Section 3 spends detailing the transformation of the integrals encountered in the region energy and its mathematical derivations leading to the Euler-Lagrange equations. Section 4 deals with the discretization and implementation on a polygonal curve. It also presents our utilization of the minimal path approach. Eventually, section 5 presents the experimental results obtained on medical and aerial images.

\section{The Deformable Generalized Cylinder}

We first recall the basics of active contours which we will rely on. The continuous active contour model is represented as a plane curve $\Gamma$ with position vector $\mathbf{c}$ :

$$
\begin{aligned}
\Gamma: \Omega & \longrightarrow \mathbb{R}^{2} \\
u & \longmapsto \mathbf{c}(u)=(x(u), y(u))^{T}
\end{aligned}
$$

where $x$ and $y$ are continuously differentiable with respect to the arbitrary parameter $u$ (different from arc length $s$ ) varying in the normalized domain $\Omega=[0,1]$. Segmentation of an object of interest is performed by finding the curve minimizing an energy functional $E$, which has the general form:

$$
E(\Gamma)=\int_{\Omega} \mathcal{L}\left(u, x, x^{\prime}, x^{\prime \prime}, y, y^{\prime}, y^{\prime \prime}\right) d u
$$

where $\mathcal{L}$ is usually made up of internal terms regularizing the curve and external terms attaching the curve to the image data. If the curve is a local minimizer of $E$, the associated Euler-Lagrange equation is verified. This implies the variational derivative of $E$ with respect to $\mathbf{c}$, which may be split over coordinates $x$ and $y$ using calculus of variations:

$$
\left\{\begin{array}{l}
\frac{\delta E}{\delta x}=\frac{\partial \mathcal{L}}{\partial x}-\frac{d}{d u} \frac{\partial \mathcal{L}}{\partial x^{\prime}}+\frac{d^{2}}{d u^{2}} \frac{\partial \mathcal{L}}{\partial x^{\prime \prime}} \\
\frac{\delta E}{\delta y}=\frac{\partial \mathcal{L}}{\partial y}-\frac{d}{d u} \frac{\partial \mathcal{L}}{\partial y^{\prime}}+\frac{d^{2}}{d u^{2}} \frac{\partial \mathcal{L}}{\partial y^{\prime \prime}}
\end{array}\right.
$$




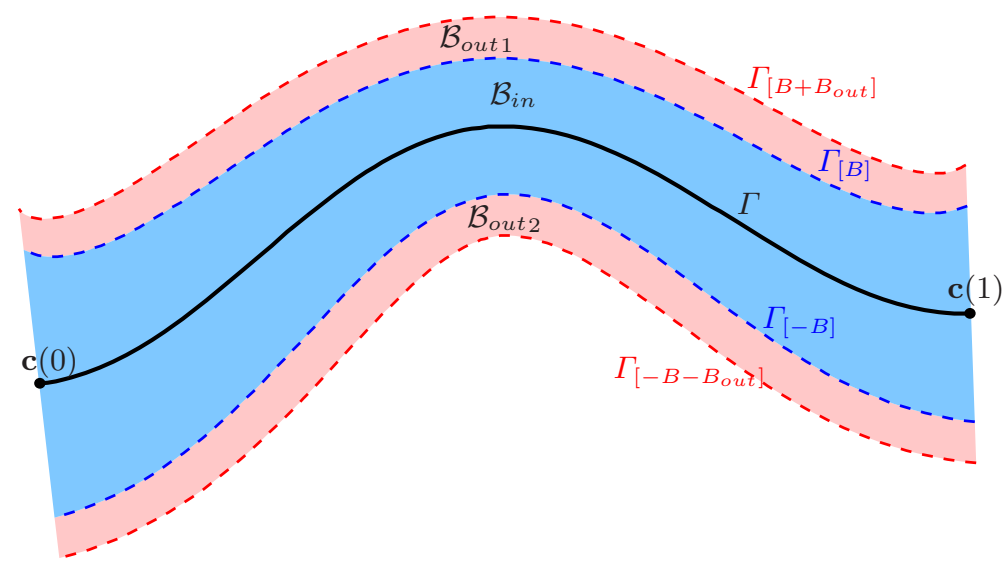

Fig. 1. Inner and outer bands for region energy

According to eq. 2 and 3 it is convenient to compute the variational derivative of $E$ when it holds only boundary integrals. This is the case with traditional gradient-based snakes [1. However, the increasing use of region terms inspired by the Mumford-Shah functional [17] has proven to overcome the limitations of uniquely gradient-based models, especially when dealing with data sets suffering from noise and lack of contrast. When dealing with region integrals, more advanced mathematical tools should be used, like Green-Riemann theorem to convert domain integrals into boundary integrals [2 18, 19, or the shape gradient method [20] 21]. When using implicit contours [22], a more practical approach consists in handling $E(\psi)$ instead of $E(\Gamma)$, where $\Gamma$ is the zero level set of $\psi$. The Euler-Lagrange equation of $E$ with respect to $\psi$ is then easily determined, like in the Chan-Vese model [6].

The deformable GC presented in this paper is region-based. Whether they are implemented using parametric contours or level sets, usual region-based deformable models are represented as closed curves, splitting the image plane into inner and outer domains. Unlike these, the GC is based on an open curve $\Gamma$ and a positive real-valued thickness function $B: \Omega \rightarrow \mathbb{R}$. The coupled curve and thickness define an inner band-shaped region $\mathcal{B}_{i n}$, whose medial axis is $\Gamma$ and width is $2 B$, as depicted in fig. 1 Region $\mathcal{B}_{i n}$ is bounded by curves $\Gamma_{[B]}$ and $\Gamma_{[-B]}$, whose respective position vectors are defined as follows:

$$
\begin{aligned}
\mathbf{c}_{[B]}(u) & =\mathbf{c}(u)+B(u) \mathbf{n}(u) \\
\mathbf{c}_{[-B]}(u) & =\mathbf{c}(u)-B(u) \mathbf{n}(u)
\end{aligned}
$$

where $\mathbf{n}$ is the oriented unit normal of $\Gamma$. One may notice that $\Gamma_{[B]}$ and $\Gamma_{[-B]}$ would be parallel curves of $\Gamma$ (also known as "offset curves" [23]) if $B$ was constant. The goal is to make both curve $\Gamma$ and thickness $B$ evolve in order to match a homogeneity criterion over $\mathcal{B}_{i n}$. Obviously, $\Gamma_{[B]}$ and $\Gamma_{[-B]}$ implicitly follow the evolution of $\Gamma$ and remain "symmetric" with respect to $\Gamma$, which 
implies a strong geometrical and topological constraint on the region. Thus, the deformable GC is dedicated to the extraction of thin shapes, like vascular structures in medical data or roads in aerial images.

The evolution of medial axis and thickness consists in determining for each boundary point $\mathbf{c}_{[B]}(u)$ (and similarly $\mathbf{c}_{[-B]}(u)$ ) whether it should move forward or backward. This decision is easier to make when an outer region is also considered, in the extent that image features at $\mathbf{c}_{[B]}(u)$ can be compared to the image statistics of both inner and outer regions. The deformable model divides the image into the objet of interest and the background, which contains usually many other objects and is therefore non-uniform. In many cases, we believe that the background is uniform only in the vicinity of the object, i.e. in narrow bands both sides apart of the ribbon. Following the principle in [15, we define two outer bands $\mathcal{B}_{\text {out } 1}$ and $\mathcal{B}_{\text {out } 2}$. We introduce a constant band thickness $B_{\text {out }}$, so that $\mathcal{B}_{\text {out } 1}$ is bounded by curves $\Gamma_{[B]}$ and $\Gamma_{\left[B+B_{\text {out }}\right]}$, as shown in fig. 11 Similarly, $\mathcal{B}_{\text {out } 2}$ is bounded by $\Gamma_{[-B]}$ and $\Gamma_{\left[-B-B_{\text {out }}\right]}$.

Rigorously, since $\Gamma$ is an open curve, position vector $\mathbf{c}$ is not differentiable at end-points $\mathbf{c}(0)$ and $\mathbf{c}(1)$, making their tangent and normal vectors undefined. From a practical point of view, we may assume that $\Gamma$ is a portion of a longer curve. As regards implementation, this requires careful discretization of derivatives using finite differences. An important property resulting from the definition in eq. 4 is that the velocity of boundary curves depends on the curvature of $\Gamma$. We denote $\ell$ the length element of curve $\Gamma$, i.e. the magnitude of its velocity vector, and $\kappa$ the curvature:

$$
\kappa=\frac{\mathbf{c}^{\prime} \times \mathbf{c}^{\prime \prime}}{\left\|\mathbf{c}^{\prime}\right\|^{3}}=\frac{x^{\prime} y^{\prime \prime}-x^{\prime \prime} y^{\prime}}{\ell^{3}}
$$

where' means differentiation with respect to $u$. The velocity vector of curve $\Gamma_{[B]}$ is expressed as a function of the velocity vector of $\Gamma$, as well as its curvature and normal. Using the identity $\mathbf{n}^{\prime}=-\kappa \mathbf{c}^{\prime}$, we have:

$$
\mathbf{c}_{[B]}^{\prime}=\mathbf{c}^{\prime}+B^{\prime} \mathbf{n}+B \mathbf{n}^{\prime}=(1-B \kappa) \mathbf{c}^{\prime}+B^{\prime} \mathbf{n}
$$

To the GC, we associate the energy functional $E$, weighted sum of the internal energy $E_{\text {smooth }}$ and the external region energy $E_{\text {region }}$ :

$$
E(\Gamma, B)=\omega E_{\text {smooth }}(\Gamma, B)+(1-\omega) E_{\text {region }}(\Gamma, B)
$$

The user-provided coefficient $\omega$ weights the influence of $E_{\text {smooth }}$ over $E_{\text {region }}$. It controls the elastic properties of the GC. Since $E$ depends both on $\Gamma$ and $B$, the GC minimizing $E$ should verify two coupled Euler-Lagrange equations:

$$
\frac{\delta E}{\delta \mathbf{c}}=\mathbf{0} \quad \frac{\delta E}{\delta B}=0
$$

The internal energy is the first-order regularizing term:

$$
E_{\text {smooth }}(\Gamma, B)=\int_{\Omega}\left\|\frac{d \mathbf{c}}{d u}\right\|^{2}+\left(\frac{d B}{d u}\right)^{2} d u
$$


Minimizing this energy encourages a regular curve and a smoothly varying band thickness. We can reasonably assume that if $\Gamma$ and $B$ are smooth, then so will be all resulting parallel curves of $\Gamma$. We define a region energy over the bands, following the work in [15]:

$$
E_{\text {region }}(\Gamma, B)=\iint_{\mathcal{B}_{\text {in }}} g_{\text {in }}(\mathbf{x}) d \mathbf{x}+\iint_{\mathcal{B}_{\text {out } 1}} g_{\text {out }_{1}}(\mathbf{x}) d \mathbf{x}+\iint_{\mathcal{B}_{\text {out } 2}} g_{\text {out }_{2}}(\mathbf{x}) d \mathbf{x}
$$

where functions $g_{\text {in }}, g_{\text {out } 1}$ and $g_{\text {out } 2}$ hold some uniformity criterion with respect to image data $I$, depending on the application. Details are given in section 4. For the moment, they should be thought of as functions penalizing non-homogeneity, the most intuitive one for grayscale images being the intensity variance, as proposed by Chan and Vese [6].

An important issue is that, as expressed in eq. 9. $E_{\text {region }}$ does not depend explicitly on position vector $\mathbf{c}$ nor band thickness $B$, making impossible the derivation leading to the Euler-Lagrange equation. In what follows, we express $E_{\text {region }}$ in a suitable form implying possible derivation and implementation on a parameterized curve discretized as a polygon. From now on, we will use the general notation $J(f, R)$ to represent the integral of function $f$ over any region $R$.

\section{Derivation of Region Energy}

In this section, we show that the domain integrals appearing in eq. 9 can be expressed as functions of $\mathbf{c}$ and $B$. The proof is based on Green-Riemann theorem, stating that for every region $\mathrm{R}$, if $(P(x, y), Q(x, y))$ is a continuously differentiable $\mathbb{R}^{2} \rightarrow \mathbb{R}^{2}$ vector field, then:

$$
\iint_{R} \frac{\partial Q}{\partial x}-\frac{\partial P}{\partial y} d x d y=\int_{\partial R} P d x+Q d y
$$

In order to apply the theorem on $J(f, R)$, we choose $P$ and $Q$ as follows:

$$
Q(x, y)=\frac{1}{2} \int_{-\infty}^{x} f(t, y) d t \quad P(x, y)=-\frac{1}{2} \int_{-\infty}^{y} f(x, t) d t
$$

The theorem expects that $\partial R$ should be at least piecewise smooth. Let us consider the more general case of a region $R$ bounded by portions of curves $\Gamma_{1}$ and $\Gamma_{2}$ and line segments $\left[\mathbf{c}_{1}\left(u_{0}\right) \mathbf{c}_{2}\left(u_{0}\right)\right]$ and $\left[\mathbf{c}_{1}\left(u_{1}\right) \mathbf{c}_{2}\left(u_{1}\right)\right]$, as depicted in fig. 2 , Starting from $\mathbf{c}_{1}\left(u_{0}\right)$ and travelling along $\partial R$ in a counter-clockwise direction, the integral of $f$ over $R$ is expressed using Green's theorem:

$$
\begin{aligned}
J(f, R) & =\int_{u_{0}}^{u_{1}} x_{1}{ }^{\prime} P\left(\mathbf{c}_{1}\right)+y_{1}{ }^{\prime} Q\left(\mathbf{c}_{1}\right) d u+\int_{0}^{1} x_{3}{ }^{\prime} P\left(\mathbf{c}_{3}\right)+y_{3}{ }^{\prime} Q\left(\mathbf{c}_{3}\right) d u \\
& +\int_{u_{1}}^{u_{0}} x_{2}{ }^{\prime} P\left(\mathbf{c}_{2}\right)+y_{2}{ }^{\prime} Q\left(\mathbf{c}_{2}\right) d u+\int_{0}^{1} x_{4}{ }^{\prime} P\left(\mathbf{c}_{4}\right)+y_{4}{ }^{\prime} Q\left(\mathbf{c}_{4}\right) d u
\end{aligned}
$$




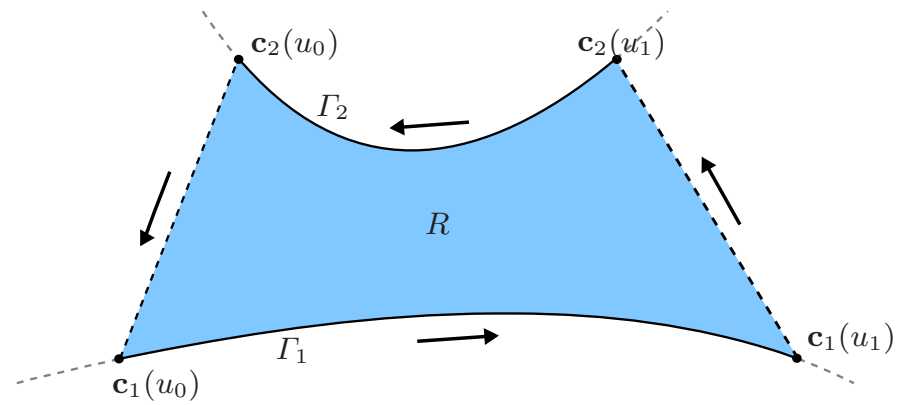

Fig. 2. Region $R$ bounded by two portions of curves $\Gamma_{1}$ and $\Gamma_{2}$. Arrows indicate counter-clockwise direction for Green's theorem.

where position vectors $\mathbf{c}_{3}=\left(x_{3}, y_{3}\right)^{T}$ and $\mathbf{c}_{4}=\left(x_{4}, y_{4}\right)^{T}$ are parameterizations of line segments $\left[\mathbf{c}_{1}\left(u_{1}\right) \mathbf{c}_{2}\left(u_{1}\right)\right]$ and $\left[\mathbf{c}_{2}\left(u_{0}\right) \mathbf{c}_{1}\left(u_{0}\right)\right]$, respectively:

$$
\mathbf{c}_{3}(u)=(1-u) \mathbf{c}_{1}\left(u_{1}\right)+u \mathbf{c}_{2}\left(u_{1}\right) \quad \mathbf{c}_{4}(u)=(1-u) \mathbf{c}_{2}\left(u_{0}\right)+u \mathbf{c}_{1}\left(u_{0}\right)
$$

Considering a family of curves $\left\{\Gamma_{\alpha}\right\}_{0 \leq \alpha \leq 1}$ interpolating from $\Gamma_{1}$ to $\Gamma_{2}$, we have:

$$
\mathbf{c}_{1}-\mathbf{c}_{2}=\int_{0}^{1} \frac{d}{d \alpha}\left\{(1-\alpha) \mathbf{c}_{2}+\alpha \mathbf{c}_{1}\right\} d \alpha
$$

The joint use of the previous relation and integration by parts in eq. 10 enables to express $J(f, R)$ directly as a function of $f, \mathbf{c}_{1}$ and $\mathbf{c}_{2}$ :

$$
J(f, R)=\int_{u_{0}}^{u_{1}} \int_{0}^{1} f\left((1-\alpha) \mathbf{c}_{2}+\alpha \mathbf{c}_{1}\right)\left(\mathbf{c}_{1}-\mathbf{c}_{2}\right) \times\left((1-\alpha) \mathbf{c}_{2}{ }^{\prime}+\alpha \mathbf{c}_{1}{ }^{\prime}\right) d \alpha d u
$$

This expression is intuitively understood since $(1-\alpha) \mathbf{c}_{2}+\alpha \mathbf{c}_{1}$ sweeps all curves between $\Gamma_{1}$ and $\Gamma_{2}$ as $\alpha$ varies from 0 to 1 . The cross product corresponds to the area of the infinitesimal quadrilaterals spanned by $\mathbf{c}_{1}-\mathbf{c}_{2}$ and $(1-\alpha) \mathbf{c}_{2}{ }^{\prime}+\alpha \mathbf{c}_{1}{ }^{\prime}$. We apply this result on our GC to calculate $J\left(f, \mathcal{B}_{i n}\right)$, replacing $\Gamma_{1}$ and $\Gamma_{2}$ with $\Gamma_{[B]}$ and $\Gamma_{[-B]}$, respectively. Introducing a variable thickness $b=B(2 \alpha-1)$, we obtain:

$$
J\left(f, \mathcal{B}_{i n}\right)=\int_{\Omega} \int_{-B}^{B} f(\mathbf{c}+b \mathbf{n}) \ell(1-b \kappa) d b d u
$$

A similar development is applied on bands $\mathcal{B}_{\text {out } 1}$ and $\mathcal{B}_{\text {out } 2}$, where $b$ varies in the intervals $\left[B(u), B(u)+B_{\text {out }}\right]$ and $\left[-B(u)-B_{\text {out }},-B(u)\right]$, respectively:

$$
\begin{aligned}
& J\left(f, \mathcal{B}_{\text {out } 1}\right)=\int_{\Omega} \int_{B(u)}^{B(u)+B_{\text {out }}} f(\mathbf{c}+b \mathbf{n})\left\|\frac{d \mathbf{c}}{d u}\right\|(1-b \kappa) d b d u \\
& J\left(f, \mathcal{B}_{\text {out } 2}\right)=\int_{\Omega} \int_{-B(u)-B_{\text {out }}}^{-B(u)} f(\mathbf{c}+b \mathbf{n})\left\|\frac{d \mathbf{c}}{d u}\right\|(1-b \kappa) d b d u
\end{aligned}
$$


The goal of these formulas is twofold. They allow to express explicitly $E_{\text {region }}$ according to $\Gamma$ and $B$, enabling the calculation of the variational derivatives. Moreover, they allow computation of $J\left(f, \mathcal{B}_{i n}\right)$ if $\Gamma$ is discretized as a polygonal line, without using a flood-filling algorithm to determine inner pixels. In order to build a gradient descent scheme, we now perform the derivation of the energies, keeping eq. 3 in mind. The differentiations of $E_{\text {smooth }}$ are straigthforward:

$$
\frac{\delta E_{\text {smooth }}}{\delta \mathbf{c}}=-2 \frac{d^{2} \mathbf{c}}{d u^{2}} \quad \frac{\delta E_{\text {smooth }}}{\delta B}=-2 \frac{d^{2} B}{d u^{2}}
$$

Regarding the region energy, the variational derivative with respect to $\mathbf{c}$ is:

$$
\frac{\delta E_{\text {region }}}{\delta \mathbf{c}}=\frac{\delta J\left(g_{\text {in }}, \mathcal{B}_{\text {in }}\right)}{\delta \mathbf{c}}+\frac{\delta J\left(g_{\text {out } 1}, \mathcal{B}_{\text {out } 1}\right)}{\delta \mathbf{c}}+\frac{\delta J\left(g_{\text {out } 2}, \mathcal{B}_{\text {out } 2}\right)}{\delta \mathbf{c}}
$$

and similarly for $B$. We found more practical to compute these derivatives over the form given by Green's theorem in eq. 10. Since $\mathbf{c}(0)$ and $\mathbf{c}(1)$ are static, they do not intervene in the derivative expression. Hence, we write:

$$
\begin{aligned}
\frac{\delta J\left(f, \mathcal{B}_{i n}\right)}{\delta \mathbf{c}} & =\frac{\delta J_{1}}{\delta \mathbf{c}}-\frac{\delta J_{2}}{\delta \mathbf{c}} \\
J_{1} & =\int_{\Omega} x_{[B]}{ }^{\prime} P\left(\mathbf{c}_{[B]}\right)+y_{[B]}{ }^{\prime} Q\left(\mathbf{c}_{[B]}\right) d u \\
J_{2} & =\int_{\Omega} x_{[-B]}{ }^{\prime} P\left(\mathbf{c}_{[-B]}\right)+y_{[-B]}{ }^{\prime} Q\left(\mathbf{c}_{[-B]}\right) d u
\end{aligned}
$$

We find that $\delta J_{1} / \delta \mathbf{c}$ has both normal and tangential components:

$$
\begin{aligned}
\frac{\delta J_{1}}{\delta \mathbf{c}} & =f\left(\mathbf{c}_{[B]}\right)\left(\ell(B \kappa-1)-\frac{B B^{\prime} \ell^{\prime}}{\ell^{2}}+\frac{B^{\prime 2}+B B^{\prime \prime}}{\ell}\right) \mathbf{n} \\
& +B B^{\prime}\left((1-B \kappa) \nabla f\left(\mathbf{c}_{[B]}\right) \cdot \mathbf{t}+B^{\prime} \nabla f\left(\mathbf{c}_{[B]}\right) \cdot \mathbf{n}\right) \mathbf{n} \\
& +f\left(\mathbf{c}_{[B]}\right) B^{\prime}(1-B \kappa) \mathbf{t}
\end{aligned}
$$

The derivative $\delta J_{2} / \delta \mathbf{c}$ is trivially obtained from eq. 15, replacing $B$ with $-B$. In the implementation part, the tangential component may be ignored, according to the principle of geodesic active contours [24], stating that the curve geometry is only affected by normal displacement whereas tangential motion only affects parameterization. We use a polygonal discretization which is resampled after each deformation step. Resampling keeps vertices almost evenly spaced along the curve and hence compensates the absence of tangential motion. The derivative with respect to $B$ is obtained from eq. 12, which does not depends on $B^{\prime}$ nor $B^{\prime \prime}$. Hence:

$$
\begin{aligned}
\frac{\delta J\left(f, \mathcal{B}_{i n}\right)}{\delta B} & =\frac{\partial}{\partial B}\left\{\int_{-B}^{B} f(\mathbf{c}+b \mathbf{n})\left\|\frac{d \mathbf{c}}{d u}\right\|(1-b \kappa) d b\right\} \\
& =\ell\left(f\left(\mathbf{c}_{[B]}\right)(1-B \kappa)+f\left(\mathbf{c}_{[-B]}\right)(1+B \kappa)\right)
\end{aligned}
$$

In the same way, the derivatives of $J\left(g_{\text {out } 1}, \mathcal{B}_{\text {out } 1}\right)$ and $J\left(g_{\text {out } 2}, \mathcal{B}_{\text {out } 2}\right)$ are calculated by replacing band thicknesses appropriately, according to eq. 13 . 


\section{Implementation}

\subsection{Polygonal Implementation}

The curve is discretized as a polygonal line made up of $n$ linked vertices $\left\{\mathbf{p}_{1}, \ldots\right.$, $\left.\mathbf{p}_{n}\right\}$. Functions $g_{\text {in }}, g_{\text {out }_{1}}$ and $g_{\text {out }}$ use global features such as area or mean intensity. Computed over $\mathcal{B}_{i n}$, these global quantities are computed according to the following template formula, which is a discrete implementation of eq. 12,

$$
J\left(f, \mathcal{B}_{i n}\right) \approx \sum_{i=1}^{n} \sum_{b=-B_{i}}^{B_{i}} f\left(\mathbf{p}_{i}+b \mathbf{n}_{i}\right) \ell_{i}\left(1-b \kappa_{i}\right)
$$

where $B_{i}, \ell_{i}, \mathbf{n}_{i}$ and $\kappa_{i}$ are the discretized band thickness, length element, curvature and normal at vertex $\mathbf{p}_{i}$, using finite differences. The same computations are performed over regions $\mathcal{B}_{\text {out } 1}$ and $\mathcal{B}_{\text {out } 2}$ by discretizing eq. 13. Vertex coordinates and corresponding thicknesses are iteratively modified using gradient descent of eq 7

$$
\begin{aligned}
\mathbf{p}_{i}^{(t+1)} & =\mathbf{p}_{i}^{(t)}-\Delta t \delta E /\left.\delta \mathbf{c}\right|_{\mathbf{c}=\mathbf{p}_{i}} \\
B_{i}^{(t+1)} & =B_{i}^{(t)}-\Delta t \delta E /\left.\delta B\right|_{B=B_{i}}
\end{aligned}
$$

The evolution of the discrete GC is sumarized by the following steps, repeated until a steady state is reached:

- Move all vertices according to eq. 17.

- Update normal vectors, areas and mean intensities

- Modify all thicknesses according to eq. 17b

- Resample the curve

- Update normal vectors, areas and mean intensities

\subsection{Initialization by Minimal Path}

Curve points $\mathbf{c}(0)$ and $\mathbf{c}(1)$, represented by vertices $\mathbf{p}_{1}$ and $\mathbf{p}_{n}$ respectively, are given by the user. They remain motionless in our approach, in the sense that the user clicks the desired end-points of the shape. Given these, the simplest way to initialize the medial curve would be a straight line. As is, the GC is sensitive to the initial location, as the energy is minimized through gradient descent. A straight line is problematic as soon as the region of interest is highly curved. The minimal path approach by Cohen and Kimmel [16] provides a reliable initialization for the central curve regardless of the shape geometry. Based on the fast marching algorithm 25] 26, this approach finds the minimal path $\mathcal{C}^{*}$ between the two end-points, given a potential-dependent metric:

$$
\mathcal{C}^{*}=\underset{\mathcal{C}}{\operatorname{argmin}}\left\{\int_{0}^{1}(w+P(\mathcal{C}(s))) d s\right\} \text { s.t. }\left\{\begin{array}{l}
\mathcal{C}(0)=\mathbf{c}(0) \\
\mathcal{C}(1)=\mathbf{c}(1)
\end{array}\right.
$$

where $s$ is the arc length parameter, $w$ is a regularizing term weighting the length and $P$ is the potential. A global search is performed, avoiding wrong 
local minima. We use the generated path as the initial guess for curve $\Gamma$, so that $\Gamma^{(0)}=\mathcal{C}^{*}$. The polygonal line $\left[\mathbf{p}_{1}, \mathbf{p}_{2}, \ldots \mathbf{p}_{n}\right]$ is generated by downsampling the path $(\approx$ a vertex every three pixels $)$. The best path between the two endpoints should be uniform and its mean intensity should be close to the one of the end-points. Hence, we chose the following potential:

$$
P(\mathbf{x})=\left(I(\mathbf{x})-\frac{I(\mathbf{c}(0))+I(\mathbf{c}(1))}{2}\right)^{2}+\|\nabla I(\mathbf{x})\|^{2}
$$

For RGB colour images, we use an extension of this potential, based on normalized RGB intensities. The end-points are intendedly kept static, so that a global minimum with respect to our criterion may be determined. Moreover, providing the end-points is an intuitive way of indicating the target path-like structure.

\section{Experiments}

We tested the proposed method on a set of images containing path-like objects of interest. First, we applied the deformable ribbon on vascular structures in grayscale medical images, mainly MRI data and angiograms. On these images in particular, we used the minimal variance principle by Chan and Vese [6]:

$$
\begin{gathered}
g_{\text {in }}(\mathbf{x})=\left(I(\mathbf{x})-\mu\left(\mathcal{B}_{\text {in }}\right)\right)^{2} \\
g_{\text {out } 1}(\mathbf{x})=\left(I(\mathbf{x})-\mu\left(\mathcal{B}_{\text {out } 1}\right)\right)^{2} \\
g_{\text {out } 2}(\mathbf{x})=\left(I(\mathbf{x})-\mu\left(\mathcal{B}_{\text {out } 2}\right)\right)^{2}
\end{gathered}
$$

where $\mu$ is the mean intensity of a given region, computed according to eq. 16. We considered a mean value for each outer region, although their respective average intensities were rather close. An important issue raised by the narrow band region energy is the similarity between inner and outer statistics that may arise when the GC, including outer bands, is initialized inside a uniform region. In such cases, we found necessary to add a bias, acting like a balloon force [27, in order to increase $B$ in proportion to the similarity between inner and outer means. Secondly, we tested the ribbon method on aerial images, in order to extract portions of rivers. On color images, we used homogeneity measures based on chromaticity, i.e. normalized RGB components. Letting $I_{\mathrm{r}}, I_{\mathrm{g}}$ and $I_{\mathrm{b}}$ be the red, green and blue intensities of a given pixel, the normalized red and green components are:

$$
\overline{I_{\mathrm{r}}}=\frac{I_{\mathrm{r}}}{I_{\mathrm{r}}+I_{\mathrm{g}}+I_{\mathrm{b}}} \quad \overline{I_{\mathrm{g}}}=\frac{I_{\mathrm{g}}}{I_{\mathrm{r}}+I_{\mathrm{g}}+I_{\mathrm{b}}}
$$

The homogeneity measure is based on the variance of intensity features, independently considered:

$$
g_{\text {in }}(\mathbf{x})=\left(\overline{I_{\mathrm{r}}}(\mathbf{x})-\overline{\mu_{\mathrm{r}}}\left(\mathcal{B}_{i n}\right)\right)^{2}+\left(\overline{I_{\mathrm{g}}}(\mathbf{x})-\overline{\mu_{\mathrm{g}}}\left(\mathcal{B}_{i n}\right)\right)^{2}
$$

where $\overline{\mu_{\mathrm{r}}}$ and $\overline{\mu_{\mathrm{g}}}$ are the average normalized red and green intensities of the corresponding region. $g_{\text {out } 1}$ and $g_{\text {out } 2}$ are expressed in a similar way. We found 
this measure to be more efficient than the L2 distance in RGB space. Indeed, normalized RGB is less sensitive to changes in illumination than can arise in a visually homogeneous region.

In order to justify the use of a region-based criterion, we provide a comparison with the same GC now endowed with an edge term, basing ourselves on the ribbon snake model of [10] and [11]. The edge-based GC is obtained by replacing the region energy with a combination of edge and balloon energies:

$$
\begin{aligned}
E_{\text {edge }}(\Gamma, B) & =\int_{\Omega} k\left(\mathbf{c}_{[B]}\right)+k\left(\mathbf{c}_{[-B]}\right) d u \\
E_{\text {balloon }}(\Gamma, B) & =\int_{\Omega} \int_{-B}^{B} \ell(1-b \kappa) d b d u=2 \int_{\Omega} B \ell d u
\end{aligned}
$$

where $k$ is an edge-detecting function (e.g. $k(\mathbf{x})=-\|\nabla I(\mathbf{x})\|$ for grayscale images). The balloon energy is the area of the inner band, obtained from eq. 12 with $f=1$. The variational derivatives, used as descent directions, were calculated as follows (note that we neglect the influence of $\Gamma$ on the balloon energy):

$$
\begin{aligned}
\frac{\delta E_{\text {edge }}}{\delta \mathbf{c}} & \approx \nabla k\left(\mathbf{c}_{[B]}\right)+\nabla k\left(\mathbf{c}_{[-B]}\right) \\
\frac{\delta E_{\text {edge }}}{\delta B} & =\left(\nabla k\left(\mathbf{c}_{[B]}\right)-\nabla k\left(\mathbf{c}_{[-B]}\right)\right) \cdot \mathbf{n} \\
\frac{\delta E_{\text {balloon }}}{\delta B} & =2 \ell
\end{aligned}
$$

The minimization of the edge energy makes the curve and thickness evolve in order to stabilize on high-gradient magnitude pixels, whereas the balloon energy results in a constant speed added to the thickness. The balloon term is weighted with a negative coefficient, in order to increase band thickness and make the GC inflate.

Results are shown in fig. 3. For each image, column (a) holds the original image. Column (b) depicts the initial configuration of the GC, used both for region and edge-based evolution. Columns (c) and (d) represents the final states of the GC, with region and edge energies respectively. In all tests, the GC exhibited strong topological and geometrical prior, preventing it from flowing into neighbouring narrow structures having more or less the same intensity/colour than the desired shape. Consequently, the deformable GC is suitable for applications where strong prior knowledge about the shape and position of the target is available. We found our region-based GC to be less sensitive to initial location than the edge-based model, as the edge energy makes the GC collapse to one side of the object in some parts. Moreover, increasing the balloon influence can make the band pass through the actual contours in other parts.

The constant outer band thickness $B_{\text {out }}$, the initial thickness $B^{(0)}$ and the internal energy weight $\omega$ are important parameters. We found that values ranging from 4 to 50 were suitable for $B_{\text {out }}$. A too small thickness may make the outer region statistics irrelevant and cause the GC boundary curves to pass through the actual boundaries, whereas the advantage of the narrow band principle is 

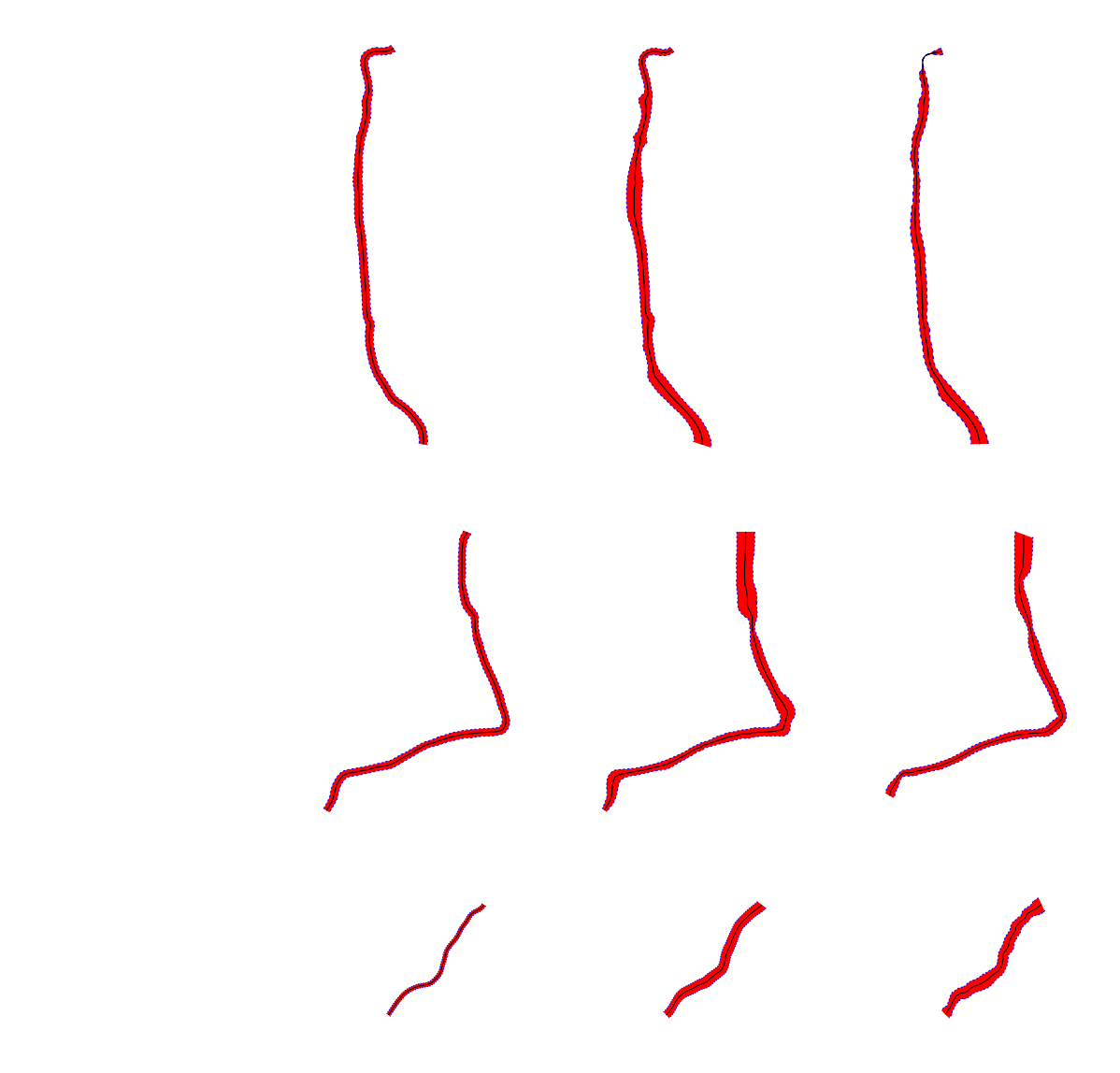

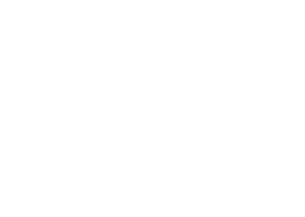

(a)

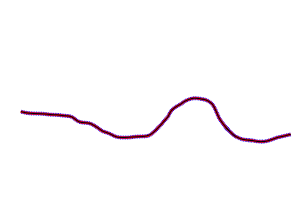

(b)

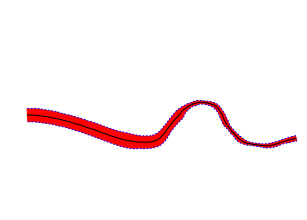

(c)

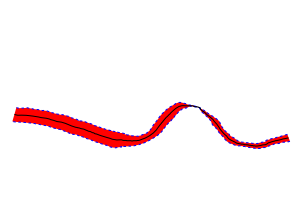

(d)

Fig. 3. Segmentation of angiograms and aerial images (c) GoogleEarth 2008): original image (a), initial GC (b), final region-based GC (c) and final edge-based GC (d)

lost if the thickness is too high. The initial inner thickness $B^{(0)}$ was typically set to 4 pixels as well. For $\omega$, values ranging from 0.4 to 0.7 were sufficient to maintain boundary curves smooth. Segmentation process on a $1000 \times 720$ RGB image (shown on the last column of fig. 3) took approximately $1.5 \mathrm{~s}$ with $\mathrm{C}++$ implementation running on an Intel Core 2 Duo $2 \mathrm{GHz}$ PC (including the computational cost due to the construction of the minimal path). 


\section{Conclusion and Perspectives}

In this paper, we presented a region-based deformable GC model, extending the work on classical region-based active contours and gradient-based ribbon snakes. Defined by a central curve playing the role of the medial axis and a variable thickness, the GC was endowed with a region-dependent energy following the narrow band principle. This energy was subsequently transformed and derived in order to allow implementation on a polygonal line deformed with gradient descent. The model proved to be efficient for recovering tubular objects, exhibiting geometrically constrained behaviour.

We may consider further investigations on this work. The deformable GC may be extended to three-dimensional space. The first 3D extension would consist in a space curve surrounded with a variable thickness, as in [28], which would be dependent on both curve parameter and angle, in order to model a deformable tube. The second 3D extension would consist in a surface homeomorphic to a plane endowed with a position-dependent thickness, which would be used to extract sheet-like structures. Substantial work has to be performed on the mathematical derivation and implementation of a suitable region energy on these 3D structures.

\section{References}

1. Kass, M., Witkin, A., Terzopoulos, D.: Snakes: active contour models. International Journal of Computer Vision 1(4), 321-331 (1988)

2. Jacob, M., Blu, T., Unser, M.: Efficient energies and algorithms for parametric snakes. IEEE Transactions on Image Processing 13(9), 1231-1244 (2004)

3. Ronfard, R.: Region based strategies for active contour models. International Journal of Computer Vision 13(2), 229-251 (1994)

4. Ivins, J., Porrill, J.: Active region models for segmenting textures and colours. Image and Vision Computing 13(5), 431-438 (1995)

5. Cohen, L., Bardinet, E., Ayache, N.: Surface reconstruction using active contour models. In: SPIE Conference on Geometric Methods in Computer Vision, San Diego, CA, USA (1993)

6. Chan, T., Vese, L.: Active contours without edges. IEEE Transactions on Image Processing 10(2), 266-277 (2001)

7. Jehan-Besson, S., Barlaud, M., Aubert, G.: DREAM2S: Deformable regions driven by an eulerian accurate minimization method for image and video segmentation. International Journal of Computer Vision 53(1), 45-70 (2003)

8. Paragios, N., Deriche, R.: Geodesic active regions and level set methods for supervised texture segmentation. International Journal of Computer Vision 46(3), 223-247 (2002)

9. Davatzikos, C., Prince, J.: An active contour model for mapping the cortex. IEEE Transactions on Medical Imaging 14(1), 65-80 (1995)

10. Mayer, H., Laptev, I., Baumgartner, A.: Multi-scale and snakes for automatic road extraction. In: Burkhardt, H.-J., Neumann, B. (eds.) ECCV 1998. LNCS, vol. 1406, pp. 720-733. Springer, Heidelberg (1998) 
11. Laptev, I., Mayer, H., Lindeberg, T., Eckstein, W., Steger, C., Baumgartner, A.: Automatic extraction of roads from aerial images based on scale space and snakes. Machine Vision and Applications 12(1), 23-31 (2000)

12. Pérez, P., Blake, A., Gangnet, M.: Jetstream: probabilistic contour extraction with particles. In: IEEE International Conference on Computer Vision (ICCV), Vancouver, Canada (2001)

13. Fua, P., Leclerc, Y.: Model driven edge detection. Machine Vision and Applications 3, 45-56 (1990)

14. Li, H., Yezzi, A.: Vessels as 4-D curves: global minimal 4-D paths to extract 3-D tubular surfaces and centerlines. IEEE Transactions on Medical Imaging 26(9), 1213-1223 (2007)

15. Mille, J., Boné, R., Makris, P., Cardot, H.: 2D and 3D deformable models with narrow band region energy. In: IEEE International Conference on Image Processing (ICIP), San Antonio, USA, pp. 57-60 (2007)

16. Cohen, L., Kimmel, R.: Global minimum for active contour models: a minimal path approach. International Journal of Computer Vision 24(1), 57-78 (1997)

17. Mumford, D., Shah, J.: Optimal approximation by piecewise smooth functions and associated variational problems. Communications on Pure and Applied Mathematics 42(5), 577-685 (1989)

18. Zhu, S., Yuille, A.: Region competition: unifying snakes, region growing, Bayes/MDL for multiband image segmentation. IEEE Transactions on Pattern Analysis and Machine Intelligence 18(9), 884-900 (1996)

19. Chakraborty, A., Staib, L., Duncan, J.: Deformable boundary finding in medical images by integrating gradient and region information. IEEE Transactions on Medical Imaging 15(6), 859-870 (1996)

20. Aubert, G., Barlaud, M., Faugeras, O., Jehan-Besson, S.: Image segmentation using active contours: calculus of variations or shape gradients? SIAM Journal on Applied Mathematics 63(6), 2128-2154 (2003)

21. Debreuve, E., Gastaud, M., Barlaud, M., Aubert, G.: Using the shape gradient for active contour segmentation: from the continuous to the discrete formulation. Journal of Mathematical Imaging and Vision 28(1), 47-66 (2007)

22. Malladi, R., Sethian, J., Vemuri, B.: Shape modeling with front propagation: a level set approach. IEEE Transactions on Pattern Analysis and Machine Intelligence 17(2), 158-175 (1995)

23. Farouki, R., Neff, C.: Analytic properties of plane offset curves. Computer Aided Geometric Design 7(1-4), 83-99 (1990)

24. Caselles, V., Kimmel, R., Sapiro, G.: Geodesic active contours. International Journal of Computer Vision 22(1), 61-79 (1997)

25. Adalsteinsson, D., Sethian, J.: A fast level set method for propagating interfaces. Journal of Computational Physics 118(2), 269-277 (1995)

26. Sethian, J.: A fast marching level set method for monotonically advancing fronts. Proceedings of the National Academy of Science 93(4), 1591-1595 (1996)

27. Cohen, L., Cohen, I.: Finite element methods for active contour models and balloons for 2D et 3D images. IEEE Transactions on Pattern Analysis and Machine Intelligence 15(11), 1131-1147 (1993)

28. Terzopoulos, D., Witkin, A., Kass, M.: Symmetry-seeking models and 3d object reconstruction. International Journal of Computer Vision 1(3), 211-221 (1987) 